\title{
Improving the Sparse Parallelization Using Semantical Information at Compile-Time*
}

\author{
Gerardo Bandera and Emilio L. Zapata \\ Department of Computer Architecture, University of Málaga, \\ P.O. Box 4114, E-29080 Málaga, Spain \\ \{bandera, ezapata\}@ac.uma.es
}

\begin{abstract}
This work presents a novel strategy for the parallelization of applications containing sparse references. Our approach is a first step to converge from the data-parallel to the automatic parallelization by taking into account the semantical relationship of vectors composing a higher-level data structure. Applying a sparse privatization and a multiloops analysis at compile-time we enhance the performance and reduce the number of extra code annotations. The building/updating of a sparse matrix at run-time is also studied in this paper, solving the problem of using pointers and some levels of indirections on the left hand side. The evaluation of the strategy has been performed on a Cray T3E with the matrix transposition algorithm, using different temporary buffers for the sparse communication.
\end{abstract}

\section{Introduction}

Research about irregular computation is presently taking more importance, though most of the parallelization techniques are only focused on dense operations. Real scientific algorithms spend the major part of its execution time in sparse matrix computations. They increment the complexity of the parallelization due to the presence of indirections. In the other hand, new algorithms contain high-level data structures, composed by several vectors. Though current compilation techniques handle all these components individually, an efficient parallelization necessitates a different approach. Hence, our first goal in this paper is to demonstrate that the performance of the SPMD code is enhanced if the semantical relationship between the data-structure components is considered.

During the last years, some works about the sparse parallelization have been developed [3/7/5]. All of these approaches intend to improve the performance by a special analysis and transformation of this part of the code. From our point of view, none of them are very efficient with real sparse algorithms, because they do not use semantic information at compile-time. Additionally, these methods are keeping away from an automatic parallelization by requiring more information from users during the compilation.

\footnotetext{
^ The work described in this paper was supported by the Ministry of Education and Culture (CICYT) of Spain under project TIC96-1125-C03.
} 
In our previous works we have demonstrated the utility of the SPARSE directive to define a sparse data-structure [101/2. With this annotation we remark the presence of semantical bindings between vectors composing the matrix, caused by the affinity of their information. To complement it, the DISTRIBUTE directive must be also included, to divide matrix entries onto the processors by means of a sparse block-cyclic distribution. The use of a pseudo-regular distribution instead of the traditional regular produces the same storage format for every local matrix than the representation of the global one.

This work describes a new feature in our compilation support: the parallelization of a run-time sparse matrix building/updating algorithm. It has a remarkable importance because compressed representations typically imply the use of pointers in code instead of coordinates. Although pointers analysis [6] grows in importance for recent applications, there are not many works addressing this problem. Our solution is based on the privatization [9], an important technique used in automatic parallelizers. The compilation strategy presented here is mainly focused on the data-parallel programming model by extending the meaning of some HPF directives. Nevertheless, we attempt to converge to the automatic parallelization of algorithms including complex data-structures by using contiguous loops analysis and pointers-to-coordinates translations. To complete our analysis, we also include a temporary storage study. It is required to optimize the performance of the sparse information communication. We will present three buffer prototypes which will be tested within the sparse transposition algorithm parallelization on a Cray T3E. The rest of the paper is divided in the following sections: Section 2 describes the compilation support for sparse readings and writings, and a sending buffer analysis for applications containing sparse communications. Section 3 includes the parallelization of an interesting case of study by using our compile-time scheme. The evaluation of our proposal will be displayed in section 4 and finally, the conclusions.

\section{Compilation Strategy Based on Privatizations}

This section describes the compile-time analysis used on the parallelization of applications containing sparse references. We present here the loops partitioning for matrix readings, its extension for sparse writings and a temporary storage selection for algorithms including sparse information interchange.

\subsection{Sparse Loops Partitioning}

Figure 1, a depicts the typical pair of nested loops accessing the non-null entries of a compressed by rows (CRS) matrix. Vector $D A$ stores the entries values and $R O$ the row pointers. $X$ is a dense vector which is being updated. Applying the owner computes rule we will obtain the SPMD code showed in figure1, b. Two additional pre-processing stages are required: for the calculation of the local bounds of the inner loop " $j "$, and for the non-local entries accessed. This alternative is the 


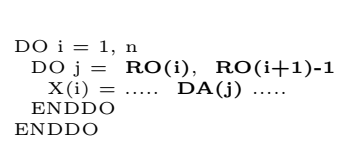

(a)

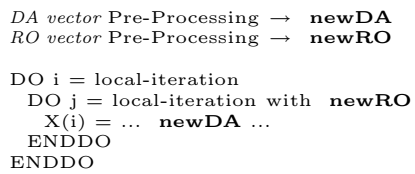

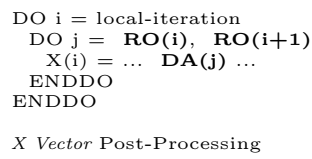

(c)

Fig. 1. (a) Sequential code reading the sparse matrix entries and updating a dense vector $X$; (b) Parallel code applying the owner computes rule to (a); (c) Parallel code after a sparse privatization: $D A$ and $R O$ vectors contain now local information.

typical inspector/executor, where two additional vectors (newRO and newDA) are filled after the loop execution by costly stages.

The local representation on each processor, caused by a pseudo-regular distribution, recommends an alternative parallelization taking into account the semantical relationship between $D A$ and $R O$ vectors. In this way, figure 1 c depicts the new parallel version of the sequential code showed in figure 1,a, where no pre-processing stages are required. This strategy, called sparse privatization, consists in the following: Every processor will only compute iterations involving its local sparse data, making private copies of remote data accessed in statements RHS, if necessary. Finally, if no locality is found, the compiler will include a post-processing stage to broadcast private results to the owner processors. With this solution we avoid costly sparse communications, trying to compute as many local operations as possible, and replacing Gather operations by Scatters.

\section{$2.2 \quad$ Sparse Matrix Updating}

Many real applications not only contain loops with sparse readings, but also includes some matrix writings (i.e, sparse addition, multiplication, transposition, LU decomposition,...). Existing parallelizations of these algorithms have two main problems: achieve a poor performance and uses complex data structures [1]. Our aim now is to extend the sparse privatization previously presented to the matrix updating in order to obtain an efficiency as close as possible to the hand-made parallel code.

Typically, the code including a sparse matrix updating is composed by some loops writing the different vectors of the matrix. Most of the related work about loops parallelization are focused to single loop partitions. However, this solution produces a poor performance with sparse updating algorithms. Therefore, our alternative analyses contiguous loops of the sequential code to detect writings on the different vectors of the matrix. After the first modification detection, the compiler will continue analyzing the remaining code to carry out a combined transformation based on the semantic information of the data-structure. It only implies the analysis of a reduced number of branches of the abstract syntax tree ( 2 or 3 at most), but the performance will be very much improved. 
As pseudo-regular distributions produce a different partition of the matrix composing vectors, it also implies a different compile-time translation of every sparse loop: (1) Pointers vector writing will produce private writings on every processor; (2) Compressed vectors updatings are also locally computed in a first step. The local pointers vectors will be used for the placement of the compressed vectors information on each processor. This compressed information will be placed on the corresponding processors in a second step.

A simplification of the parallelization process is depicted in figure 2 Note that this transformation will be completed when the data vector $(D A)$ modification had been detected. This is not a mandatory, but an important percentage of real codes follow this requirement. Nevertheless, a generic parallelization process is also included in our compilation support, with a more costly semantical dependence analysis. As readers can observe, the parallelization of loops containing a coordinates vector modification $(R O$ and $C O$ ) is done by creating private copies. This local information must be stored on temporary buffers, which will be sent afterwards to the appropriate destinations. The $D A$ updating detection implies the inclusion of two additional routines within the SPMD code: a Communication and a Matrix Reconstruction. If the data modification is not detected in the following loops, the parallelization will be completed only with new coordinates. The number of loops to analyze after the $C O$ and $R O$ updating will depend on an input parameter of the parallelization tool, which will be fixed at compile-time.

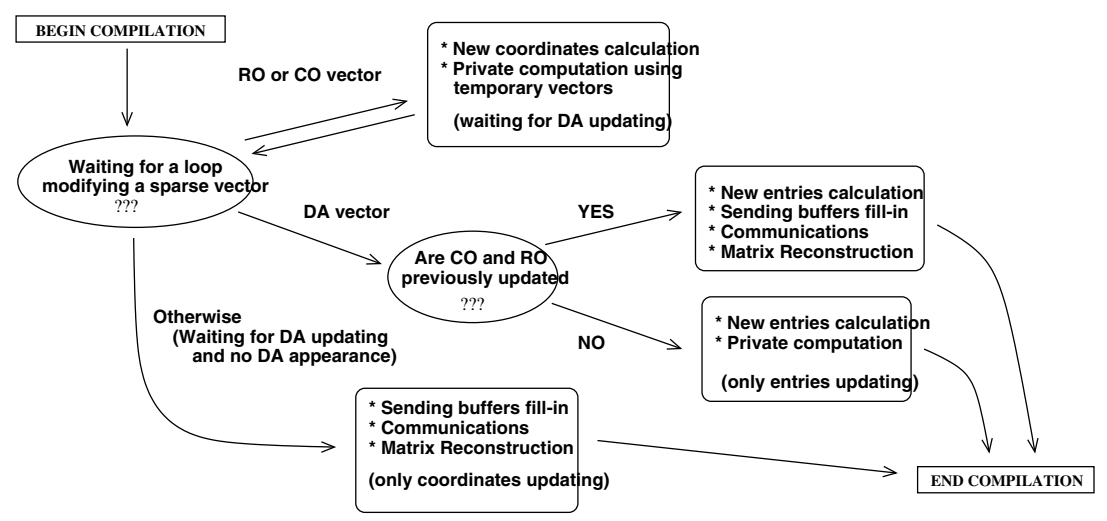

Fig. 2. Compiler strategy for algorithms containing a sparse matrix updating.

\subsection{Buffering Analysis}

A temporary storage study is necessary when the application to parallelize requires sparse data interchange between processors. The selection of an efficient data-structure to allocate the information involved in the communication has 
a remarkable influence in the parallel performance: first, in the Collecting and Mixing stages, with a different index processing; and second, in the Communication time, with the necessity of an implicit coordinates storage. Hence, we have implemented the three following alternatives for the sparse data interchange: the Unsorted Buffer, the Linked-Lists and the Histogram Buffer.

In the Unsorted Buffer, source processors pack the matrix entries in the same order they are visited. As it does not typically coincide with the order in destination, an explicit inclusion of coordinates will be required for the matrix reconstruction. The memory occupation of this buffer only depends on the maximum number of elements to send to a single processor. As this value is not known at compile-time, a good estimation is required to avoid a memory overhead. Linked-Lists are based on dynamic memory allocations and pointers arrangements. Every data entry is stored in a cell with one of the coordinates, while the second one is used to select the list where this cell will be linked to. The number of cells on a given list indicates the non-nulls of this row. In this buffer the cell allocation is done by demand, so the memory reservation will be minimal and no estimation is required. The Histogram Buffer is also composed by three vectors. The first two store in a sorted fashion data entries and one coordinate, while a third vector contains counters of elements belonging to the same dimension. While the length of this last vector coincides with the number of rows in destination, the first two are divided in slices of the same size, where the elements belonging to the same row are placed. A careful slices size estimation is needed, because the different occupation percentage of every row can produce many memory wasting.

\section{Parallelization of the Matrix Transposition}

This section describes the parallelization of the sparse transposition algorithm using our compile-time strategy. The selected code is a very motivating example containing three level of indirections in some statement LHS. The data-parallel version of the transposition is based on the sequential code developed by Pissanetzky in [8]. This is the most efficient sequential algorithm, in spite of its strange code structure. There exists a second alternative for this algorithm, which is simpler to understand but performs worse. The HPF code is shown in figure 3. We declare a $N \times M$ CRS sparse matrix $A$ with vectors ( $D A, C O$ and $R O)$ and alpha non-null entries. The transposed matrix newA is also defined in a similar way using another triplet of vectors. With the DISTRIBUTE and the first $A L I G N$ directive, we are specifying a SPARSE-CYCLIC $(\mathrm{k})$ distribution for both matrices. The second alignment is for the dense vector $R O W 2$. It is used as an extra pointers vector to avoid costly memory occupations and an additional classification step. We have extended the meaning of this directive. When a dense vector is aligned with a pointers vector of a sparse matrix, we specify the same distribution for both the alignee and the target of the alignment. Thus, they will only have a local meaning. 

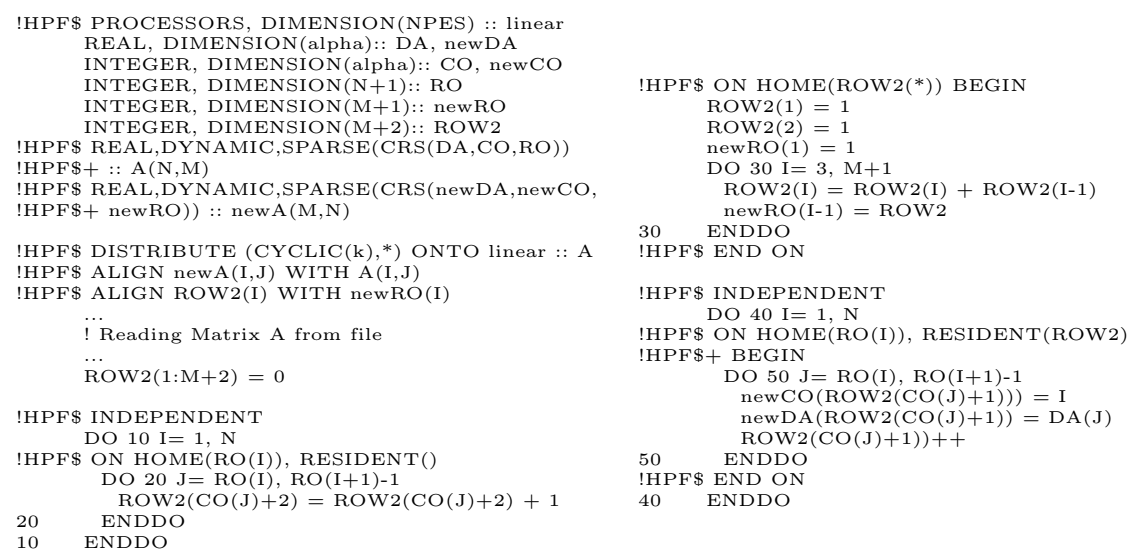

Fig. 3. HPF Sparse Matrix Transposition.

The data-parallel code can be decomposed in two main parts: the pointers vector of the new matrix (newRO) is calculated in the first part of the code, while $n e w D A$ and newCO are filled in the second. The first statement after the file reading is parallel in fact, because it is written using Fortran90. The partition of loop 10-20 is driven by the INDEPENDENT and ON HOME annotations. They indicate a parallel execution on each processor using their local submatrices, and also obtaining private results. In the next part of the code, together with the $O N H O M E$ directive, writings on pointers vectors also indicates this privacy. Thereby, every processor will use and calculate private values of vectors $R O W 2$ and newRO.

The last part of the algorithm is the data and column vectors updating (loops 40-50). At this moment of the compilation, the pointers vector of the new matrix has being already modified. In the same way that loops $10-20$, the INDEPENDENT and ON HOME directives cause a sparse privatization, where every processor will compute a set of iterations with its local submatrices. As we have depicted in figure 2 the newDA writing causes the completion of the matrix updating. Hence, the compiler will include a Collecting stage in order to fill the sending buffers in. Moreover, a Communication stage and a final matrix reconstruction (Mixing) will be included after the loop execution. For this last part of the parallelization, the compiler must select one of the three buffering alternatives presented in section 2.3

By analyzing the different parts of the HPF code, we have deduced that some annotations can be removed. The main requirements of our compilation support are the declaration part annotations: the SPARSE directive, because it defines the semantic relationship between the different vectors composing the matrix; and the DISTRIBUTE and ALIGN directives, specifying the owner processors of every matrix entry. Two main details of this concrete application make sure its automatic parallelization: (1) The loops bounds: from the above directives the 
compiler knows that loops 10-20 and 40-50 are used to visit the matrix $A$ by rows, and thus, every processor will execute different loops iterations only with its local submatrices (sparse privatization); (2) The LHS vectors: while pointers vectors writings imply private computation, data vector updating require the completion of the transformation, including Collecting, Communication and Mixing stages.

\section{Experimental Results}

In this section we evaluate the efficiency of our compilation with the case of study presented in section 3. We have used the Cray T3E, SHMEM routines and the $c c$ compiler with the $-\mathrm{O} 2$ turned on. We have tested different matrices and distribution parameters, but we only include here results for two large matrices from the Harwell-Boeing Collection: a very sparse matrix (BCSSTK30 or B30) containing 1036208 non-nulls and with 28924 rows and columns (density rate $=0.12 \%$ ), and a very dense sparse one (PSMIGR1 or PS1), with order 3140 and 543162 entries $(5.51 \%)$.

The first evaluation is about the influence of the sending buffer in the performance of the transposition. Figure 4 shows the total time of the algorithm for the three buffering schemes previously described. As we can observe, the best performance version uses the Histogram Buffer, because of the nice cache behavior of the sorted information. Although the memory occupation is lesser, the necessity of sorting the entries at destination produces an important delay when using the Unsorted Buffer. Finally, the worst buffer selection is the Linked Lists, where the code overhead is incremented with the idle time produced by continuous cells allocations. Nevertheless, this alternative is the only one useful with very large matrices. Buffers enhancements are more remarked for dense matrices, because the number elements to store on every dimension grows.
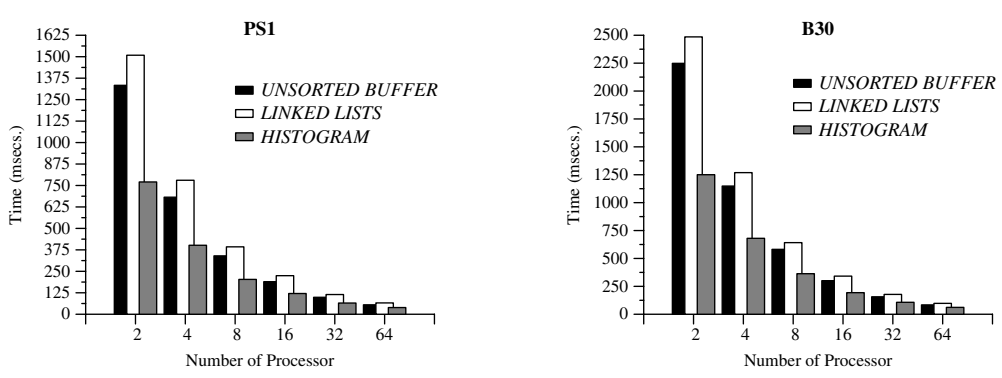

Fig. 4. Execution time with every buffering alternative. PS1 and B30 matrices.

A different way of testing the powerful of our compilation strategy is performing a comparison with the typical run-time support using pre and post-processing stages for code indirections. Previous works [10,1] have illustrated the benefits 
of similar approaches to ours in comparison with CHAOS[11] for matrix readings. For sparse writings, the resulting code with CHAOS increments the delay, because it will need many more pre-processing stages. The expected results with PILAR 4] are very similar, because even improving the CHAOS performance, the sparse relationship between the dense vectors composing the matrix has not been taken into account. For the same reason, our approach also increments the performance regarding to traditional sparse solvers. The excellent scalability of the translated code must be underlined, in spite of transposition mainly performs data movements. In the same way, we have also obtained an efficient parallelization, because the time of the sequential version (370.71 msec. for PS1 and 231.59 msec. for B30) is improved from 8 and 16 processors, respectively.

\section{Conclusions}

Sparse references increment the complexity of the parallelization, due to the presence of many code indirections and a replacement of coordinates by pointer values. In the other hand, many sparse applications present computation locality, which can be exploited by providing information to the compiler about the structure and the data distribution. This information is enough to improve the parallel performance. The parallelization support presented in this work is based on the semantical relationship of the different vectors composing a highlevel data-structure. It is denoted by the SPARSE directive. This one, jointly with the use of a pseudo-regular distribution, implies the replacement of the owner computes rule by a sparse privatization approach, where the computing processor is the sparse entries owner. At the same time, a multi-loop analysis is also enabled. With our solution, costly pre-processing stages and sparse communications are removed.

The dynamic sparse building/updating has also been addressed in this paper. Our compilation algorithm has been described and tested with a remarked application: the transposition. Parallel codes containing sparse communications also require a buffering study. We have presented here three alternatives for storing data entries and coordinates. They will be useful depending on the memory limitations. Although the parallelization approach presented here is based on a sequential code annotation, it constitutes a first step to the automatic parallelization of applications containing high-level data structures.

\section{References}

1. R. Asenjo. LU Sparse Matrices Factorization on Multiprocessors. PhD thesis, Computer Architecture Dept., University of Málaga, 1997.

2. G. Bandera. Semi-Automatic Parallelization of Applications containing Sparse Matrices. PhD thesis, Computer Architecture Dept, University of Málaga, 1999.

3. A.J.C. Bik. Compiler Support for Sparse Matrix Computations. PhD thesis, University of Leiden, The Netherlands, 1996.

4. D.R. Chakrabarti, N. Shenoy, A. Choudhary, and P. Banerjee. An efficient uniform run-time scheme for mixed regular-irregular applications. In Proc. of ICS'98. 
5. F. Delaplace and R. Adle. Extension of the dependence analysis for sparse computation. In Proc. of Parallel and Distributed Computing Systems, October 1997.

6. R. Ghiya and L.J. Hendren. Putting pointer analysis to work. In Proc. of the $25^{\text {th }}$ ACM SIGPLAN-SIGACT Symp. on Principles of Programming Languages, 1998.

7. V. Kotlyar, K. Pingali, and P. Stodghill. Compiling parallel code for sparse matrix applications. In Proc. of Supercomputing, 1997.

8. S. Pissanetzky. Sparse Matrix Technology. Academic Press Inc., 1984.

9. P. Tu and David Padua. Automatic array privatization. Sixth Workshop on Languages and Compilers for Parallel Computing, 1993.

10. M. Ujaldón, E.L. Zapata, B. Chapman, and H.P. Zima. Vienna-Fortran/HPF extensions for sparse and irregular problems and their compilation. IEEE Trans. on Parallel and Distributed Systems, 8(10):1068-1083, 1997.

11. J. Wu, R. Das, J. Saltz, and H. Berryman. Distributed memory compiler design for sparse problems. IEEE Trans. on Computers, 44(6):737-753, June 1995. 